\title{
Leben zuerst
}

\section{Das Arbeitsleben mit Lebensarbeit verbinden und Verwirklichungschancen mehren}

\author{
Wolf Rainer Wendt
}

Prof. Dr. Wolf Rainer Wendt ist

Vorsitzender der Deutschen Gesellschaft für Care und Case Management sowie Honorarprofessor der Eberhard Karls Universität Tübingen. Er ist seit fast vier Jahrzehnten Mitglied im Beirat der Blätter der Wohlfahrtspflege.

E-Mailwendt@imails.de
Es gibt ein Leben neben der Erwerbsarbeit. Leistungen in Familie, freier Betätigung oder Verein müssen bei der Eingliederung in Arbeit berücksichtigt werden. Ein beschäftigungsorientiertes Fallmanagement verlangt nach einem ChancenManagement unter Anerkennung der nichtentgeltlichen Leistungen außerhalb des traditionellen Arbeitsmarktes.

Grundsicherung schließt für Erwerbsfähige die Beschäftigungsförderung ein. Institutionell ist mit der Verbindung von Sozialhilfe und Arbeitslosenhilfe ein Übergang von Dienstleistungen am Arbeitsmarkt in »Dienste am Menschen « vollzogen worden, womit nun die ganze Lebenssituation von Hilfesuchenden bei ihrer Integration in Arbeit in Betracht zu ziehen und zum Gegenstand der Beratung und Unterstützung zu machen ist.

Zur Beschäftigungsförderung muss nach Arbeit gesucht werden. In der »Hilfe zur Arbeit « wird gemäß dem Prinzip des Forderns der Arbeitslose dazu angehalten. Sache eines Managements der Versorgung mit Arbeit ist aber dem Prinzip des Förderns nach, die Chancen auf Beschäftigung gerade für die Menschen zu mehren, die bei eigener Suche wenig Erfolg haben. Mit Arbeitsanreizen ist nichts gewonnen ohne reale Arbeitsmöglichkeiten. Ein beschäftigungsorientiertes Fallmanagement, so die These dieses Beitrags, verlangt nach einem Chancen-Management im System der Beschäftigung bei Anerkennung der nichtentgeltlichen Leistungen außerhalb von ihm und mit der Gestaltung von Übergängen zwischen Lebensarbeit und Arbeitsleben.

\section{Ein Prozess der Begleitung: internationale Beispiele}

Eingliederung in Arbeit ist ein Prozess wechselseitiger Anpassung von Möglichkeiten der Beschäftigung und Potenzialen einer Person. In der Beschäftigungsförderung kann auf eine weitgehende internationale Übereinstimmung in der Anwendung des Prinzips »Fördern und fordern « verwiesen werden:

- Seit 1998 gibt es in Großbritannien die New-Deal-Programme »welfare-towork $«$.

- In Frankreich gehören seit 2001 zum institutionellen Hilfeplan zur Rückkehr ins Arbeitsleben (»Plan d'aide au retour à l'emploi «, PARE) ein Assessment, in dem die Wünsche und Fähigkeiten des Antragstellers erkundet werden, und die Erstellung eines persönlichen Handlungsplans (»Projet d'action personalisée«, PAP) sowie bei Arbeitslosen, die eine intensive Begleitung nötig haben, ein »Contrat d'accompagnement «.

- Eine individualisierte Wiedereingliederung Arbeitsloser wird analog in den Niederlanden betrieben. Im Intake-Bereich der niederländischen Zentren für Arbeit und Einkommen (CWI, die für die deutschen Jobcenter das Vorbild abgegeben haben) teilt man die Arbeitsuchenden einer von vier ProfilingKlassen zu, wonach die schwerer vermittelbaren Personen von Case Managern betreut werden, die private Dienstleister zur Reintegration ihrer Klienten heranziehen können. »Trajectbegeleiding « heißt im Niederländischen das schrittweise Vorgehen in der Integrationsarbeit, indem konkrete, aufeinander aufbauende Zielvereinbarungen getroffen und schrittweise Hilfen geplant werden. In Dänemark wird für Erwerbslose ein individueller Aktionsplan entwickelt, der ebenfalls die mitgebrachten Befähigungen und persönlichen Wünschen mit den Gegebenheiten des Arbeitsmarktes abgleicht.

- In Australien hat man in der Welfareto-work-Politik des letzten Jahrzehnts eine Abfolge der Unterstützung organisiert, die mit der Meldung bei dem für jede Art Sozialhilfe zuständigen Multi- 
service-Agentur der Regierung »Centrelink « beginnt, dann zum »Job Network « führte, wo man für Klienten, die länger als zwölf Monate arbeitslos sind, ein Case Management in Form einer »Intensive Support Customized Assistance « bereithielt. Sind Eingliederungshemmnisse wie Wohnungslosigkeit, psychische Erkrankung, Drogenprobleme, Schulden, soziale Isolation oder andere soziale Probleme vorhanden, so setzte ein »Personal Support

Rahmen seiner persönlichen Daseinsvorsorge erfolgt, seine Hilfe zur Selbsthilfe bei besonderen Belastungen des Lebens eingeschlossen, dürfte die Förderung dann individuell angemessen sein, wenn sie auf diese zweckmäßige Selbsttätigkeit in der jeweiligen Lebenssituation abgestimmt wird. In ihr ist der Horizont erschlossen, in dem der Einsatz einer Person erwartet werden kann. Sie handelt im Rahmen von Verwirklichungsmöglichkeiten (capabilities), die sie für sich er-

\section{"Das System der Beschäftigung braucht ein Chancen-Management"}

Programme « (PSP) ein, das mit Beratung, Training, Therapie und anwaltlicher Begleitung eine Brücke zwischen »crisis assistance « und »employment assistance programme" schlug (siehe hierzu Muller/Galton 2006, Perkins 2007). Seit Juli 2009 ist das » Job Network « durch »Job Services Australia « ersetzt und das Angebot neu gestaltet worden. Für jeden Arbeitsuchenden ist nun ein »Employment Pathway Plan « vorgesehen, der nach Zuweisung zu einer von vier Bedarfsgruppen in abgestufter Intensität verfolgt wird.

In Deutschland umfasst die Grundsicherung für Arbeitsuchende nach $\mathbb{\int} 1$ Abs. 2 SGB II »Leistungen 1. zur Beendigung oder Verringerung der Hilfebedürftigkeit insbesondere durch Eingliederung in $\mathrm{Ar}$ beit und 2. zur Sicherung des Lebensunterhalts «. Gestärkt werden soll die Eigenverantwortung; das Gesetz fordert von den Empfängern der Leistungen eine Gegenleistung, nämlich das ihrerseits Mögliche zu tun, um die Hilfebedürftigkeit zu beenden. Vorrangig dienen die gebotenen Leistungen der unmittelbaren Aufnahme einer Erwerbstätigkeit. Stehen Hindernisse einer direkten Eingliederung im Wege, ist nach dem Prinzip des Förderns eine "umfassende Unterstützung mit dem Ziel der Eingliederung in Arbeit « geboten.

$\mathrm{Zu}$ fragen ist nun nach dem Gegenstand der individuellen Beschäftigungsförderung, mit deren Maßnahmen im Sinne des $\mathbb{1} 1$ SGB I der Erwerb des Lebensunterhalts durch eine frei gewählte Tätigkeit ermöglicht werden soll. Da die Gegenleistung des Hilfebedürftigen im kennt. Das heißt, ihre Erwerbs- und Beschäftigungsfähigkeit und ihre Arbeitsbereitschaft bestehen im Kontext von Chancen, die sich individuell wahrnehmen und realisieren lassen.

Beschäftigungsförderung verspricht fallweise Erfolg, wenn sie solche Chancen erschließt und entwickelt. Insbesondere bei Personen und für Bedarfsgemeinschaften, denen ihre Lage und korrespondierend die Lage am Arbeitsmarkt nicht hinreichend Chancen zur Integration in Arbeit geben. Sie sind in den Möglichkeiten ihres Lebens eingeschränkt. Integration nachhaltig fördern heißt, so die These, statt des Prinzips "work-first " den Grundsatz »life-first " gelten lassen (Dean et al. 2005, 3).

\section{Produktive Teilhabe einräumen}

Lebenstätigkeit geht der Erwerbstätigkeit vor. Auch ohne einen festen Arbeitsplatz gibt es viel zu tun. Wer in das gesellschaftliche Leben wenig und ins Beschäftigungssystem gar nicht integriert ist, muss umso mehr alleine zurechtkommen. Es fehlt ihm an Teilhabe; bei Einsicht in seine beschränkten Möglichkeiten sucht er mit ihnen existenziell auszukommen. Je »marktferner « jemand situiert ist, desto mehr ist mit seiner Autonomie zu rechnen. Als Langzeitarbeitsloser hat er sich wohl oder übel auf ein anderes Leben einrichten müssen. Es mag ein von Passivität geprägtes Leben sein, ist sehr viel öfter aber auch in der Krise der Arbeitslosigkeit und bei Vermittlungshemmnissen ein mit Aktivitäten ausgelastetes Leben.
Bekanntlich verweilt die Personengruppe der Alleinerziehenden am längsten im Bezug von Leistungen nach SGB II und bei ihr ist auch das Armutsrisiko am größten. Vor allem Kinderbetreuungsprobleme verhindern die Aufnahme einer bedarfsdeckenden Erwerbstätigkeit. (Möller u.a. 2009, 7, vgl. Bundesagentur 2008) Die Bereitschaft, den Lebensunterhalt durch Erwerbstätigkeit zu sichern, ist bei den Alleinerziehenden nicht geringer als bei anderen Personengruppen, aber die Angebotsstruktur steht ihr entgegen. Es fehlt an der Abstimmung von zeitlichen und örtlichen Konditionen eines Arbeitsplatzes auf die Situation einer alleinerziehenden Mutter mit dem Kind oder den Kindern. Nun ist die familiäre Erziehungsleistung eine Lebens- und Arbeitsleistung, die bei Kindern unter drei Jahren voll anerkannt wird, aber auch bei Kindern über drei Jahren hoch zu schätzen ist und bei Ausfall den Einsatz einer Menge formeller fachlicher Beschäftigung nach sich zieht. Die Anerkennung jener Leistung sollte in jedem Fall Voraussetzung einer auf Überwindung der Hilfebedürftigkeit gerichteten Beratung sein.

Vereinbarkeit von Familie und Beruf wird in der demografischen Krise überall verlangt; sie kann aber auch eine Balance von Leben und Arbeit derart bedeuten, dass Erwerbstätigkeit in weit größerem Maße als bisher mit der persönlichen Lebenstätigkeit verwoben wird. Bei Hochqualifizierten geschieht das oft schon, weil die Kreativität eines Beschäftigten in zeitlicher und örtlicher Beweglichkeit am besten genutzt wird. Geringqualifizierten kann damit geholfen werden, dass das Spektrum ihrer Einsatzmöglichkeiten erweitert wird und ein Zuschnitt auf die individuelle Lebenssituation erfolgt. Voraussetzung ist, dass von vorhandenen Stärken ausgegangen und die persönliche Lage respektiert wird, damit sich die Organisation ihres Lebens mit der Organisation ihrer Arbeit vereinbaren lässt.

\section{Verbreiterung der Arbeitsförderung}

Der monetäre Leistungsbezug im Rechtskreis des SGB II ist auf den Haushalt einer Person und Bedarfsgemeinschaft bezogen. Als passive Förderung erfolgt er zur materiellen Existenzsicherung. Die Bedürftigkeit besteht auch, wenn (bei 
unzureichendem Erwerb) gearbeitet wird. Die aktive Beschäftigungsförderung beschränkt sich deshalb nicht darauf, Arbeit zu vermitteln; sie ist frei, sich in ihrer Art und Weise auf die Umstände und Möglichkeiten individueller Lebensführung einzustellen. Sie kann selbstaktive Versorgung, Eigenarbeit, Lernarbeit, Bemühungen um physische und psychische Rehabilitation usw. berücksichtigen - und damit personenbezogen Verbindungen von Lebensarbeit und Arbeitsleben herstellen.

Die stärkere Verknüpfung der Beschäftigungsförderung von Arbeitssuchenden nach SGB II mit der Arbeitsförderung nach SGB III, wie sie das im Januar 2009 in Kraft getretene Gesetz zur Neuausrichtung der arbeitsmarktpolitischen Instrumente intendiert, erlaubt ein flexibleres Regime des Übergangs in der Teilhabe an Arbeit. In Hinblick auf Erziehung und Pflege stellt $\ 8$ Abs. 1 SGB III auf die Vereinbarkeit von Familie und Beruf ab: »Die Leistungen der aktiven Arbeitsförderung sollen in ihrer zeitlichen, inhaltlichen und organisatorischen Ausgestaltung die Lebensverhältnisse von Frauen und Männern berücksichtigen, die aufsichtsbedürftige Kinder betreuen und erziehen oder pflegebedürftige Angehörige betreuen oder nach diesen Zeiten wieder in die Erwerbstätigkeit zurückkehren wollen. «

Was für die genannten Personengruppen gilt, lässt sich auf andere Lebensleistungen ausdehnen. So kann man dem Kompetenzprofil älterer Arbeitsuchender mit darauf zugeschnittenen Beschäftigungsverhältnissen entsprechen. Die Aufgabe einer aktiven Arbeitsförderung besteht danach in einem Chancen-Management im (lokalen) Beschäftigungssystem in Rücksicht auf individuelle Verwirklichungsmöglichkeiten in einem breiten Spektrum von informeller und formeller Betätigung.

Es gibt längst einen breiten volkswirtschaftlichen Diskurs über die Einbeziehung sozialer Aktivitäten in das ökonomische Geschehen und seine Performanz (siehe zuletzt den Bericht der Commission on the Measurement of Economic Performance and Social Progress von Stiglitz, Sen und Fitoussi, September 2009). Wird anerkannt, dass im Familienhaushalt, in der Selbstversorgung wie in freiwilligen sozialem Engagement wertschöpfend gearbeitet wird, kann die Grundsicherung für Arbeitsuchende in ihrer Beziehung auf Eigenverantwortung mehr sein als ein
Ausfallbürge. Sie gewinnt eine Wohlfahrt stiftende Funktion. Es geht dann bei der Eingliederung ins Erwerbsleben nicht um "Arbeit um jeden Preis ", sondern um die Gestaltung von Teilhabe.

»Partizipation statt Protektion « war in den Niederlanden die Devise bei der Eingliederung von Arbeitslosen bereits in den 1990er Jahren. Die angestrebte Teilhabe erforderte eine soziale Aktivierung auf den Weg über Angebote unbezahlter Beschäftigung. (Van Berkel/Coenen/Dekker 1999, 101 ff.) Diese Angebote bieten naturgemäß keine vollständige Teilhabe am Arbeitsleben, aber empirisch bewähren sie sich in vielen Fällen in einem Regime des Übergangs. Teilnehmer an den niederländischen Maßnahmen berichten, dass ihnen Freiwilligenarbeit die Erfahrung vermittelt, für die Gesellschaft nützlich zu sein, anderen Menschen helfen zu können und als Partner in sozialen Beziehungen respektiert zu werden. Ihr sozialer Horizont und ihre soziale Vernetzung erweitern sich und sie gewinnen an Kompetenz. (Van Berkel/Coenen/Dekker 1999, 104)

Leistungsanforderungen und soziale Einbindung bedingen einander dort, wo im Einsatz von Arbeitsuchenden ihre Potenziale gefragt sind und entwickelt werden. Mit ihren spezifischen Organisationsformen kann die Sozialwirtschaft viel zur Gestaltung von Übergängen beitragen, insbesondere für »arbeitsmarktferne« Personengruppen.

\section{Lebensbewältigung und Arbeitsbefähigung verbinden}

Es gibt verschiedene Weisen der Teilhabe an Arbeit, Ausbildung und lebenslangem Lernen und sie lassen sich auf vielfältige Art und Weise kombinieren. Ohne Zweifel muss der Einzelne im Wandel der Anforderungen ständig aktiv sein, um Befähigung zu erwerben und zu erhalten.

Bei jungen Menschen lassen die Jugendkompetenzagenturen erkennen, was eine breite Förderung lebenspraktischen Wissens und Könnens und ein Training der Alltagsbewältigung zur Beschäftigungsfähigkeit (employability) beizutragen vermag. Benachteiligte und bildungsferne Personengruppen erhalten Verwirklichungschancen durch eine Qualifizierung ihrer gewöhnlichen Lebensführung als einer Aneignungsweise, in der sie auch erwerbstätig werden können.
Zum Arbeitsvermögen tragen alle Dimensionen des Lebens bei; ihnen muss Raum gegeben, Übung erlaubt und Eigensinn gestattet sein. Verwirklichungschancen (capabilities) sind nach Amartya Sen Befähigungen im Sinne von substantiellen Freiheiten eines Menschen, das zu tun, was er nach seiner Wertschätzung zu seinem Wohlergehen tun will. So fängt Eingliederung in Erwerbsarbeit individuell bei der Sorge um die eigene Entwicklung an, und die Möglichkeiten der Integration wachsen auf Wegen über soziale Aktivierung.

Die Anerkennung von Erziehung und Pflege als Arbeit ist ein Schritt über die Grenze zwischen Arbeitsleben und Lebensarbeit. Mehr noch verbinden sie sich in einer » Tätigkeitsgesellschaft « mit der Wertschätzung vielfältiger sozialer und bildender Aktivitäten vor, neben und zwischen formellen Beschäftigungsverhältnissen - mit der Chance, dass diese sich an ihrer Basis vermehren.

\section{Literatur}

Bundesagentur für Arbeit, Arbeitsmarktberichterstattung: Alleinerziehende im SGB II. Nürnberg 2008.

Dean, Hartley/Bonvin, JeanMichel/Vielle, Pascale/Farvaque, Nicolas: Developing capabilities and rights in welfare-to-work policies. In: European Societies, 7, 1, 2005. S. 3-26.

Möller, Joachim u. a.: Der Arbeitsmarkt hat profitiert. IAB-Kurzbericht 29/2009. Institut für Arbeitsmarkt- und Berufsforschung, Nürnberg 2009.

Muller, Gerda A./Galton, Celeste: The Personal Support Programme: »Where we walk the walk «. In. The Australian Community Psychologist, 18, 3, 2006. S. 43-49.

Perkins, Daniel: Improving Employment Participation for Welfare Recipients Facing Personal Barriers. In: Social Policy and Society, 7, 1, 2007. S. 13-26.

Van Berkel, Rik/Coenen, Harry/Dekker, Arjen: Regulating the unemployed: From protection to participation. In: Lind, Jens/Møller, Iver Hornemann (eds.): Inclusion and Exclusion: Unemployment and Non-standard Employment in Europe. Ashgate, Aldershot 1999. S. 89-109. 